Discussion Paper No. 827

\title{
CONFORMISM AND STRUCTURAL CHANGE
}

\author{
Takeo Hori \\ Masako Ikefuji \\ Kazuo Mino
}

December 2011

The Institute of Social and Economic Research Osaka University

6-1 Mihogaoka, Ibaraki, Osaka 567-0047, Japan 


\title{
Conformism and Structural Change*
}

\author{
Takeo Hori ${ }^{\dagger}$ Masako Ikefuji ${ }^{\ddagger}$ and Kazuo Mino ${ }^{\S}$
}

December 27, 2011

\begin{abstract}
We study structural change in a simple, two-sector endogenous growth model and show that the presence of commodity-specific consumption externalities can be a source of structural change. When the degrees of consumption externalities are different between different goods, the two sectors grow at different rates, whereas the aggregate economy exhibits balanced growth in the sense that capital stock and expenditure grow at the same constant rate. Under the more restrictive condition such that the degrees of consumption externalities are the same, structural change does not occur. We also show that the dependence of the benchmark consumption levels on the past consumption is crucial for the divergent patterns of structural change across countries.
\end{abstract}

Keywords: Structural change, Consumption externalities, Two-sector growth model, Kaldor facts

JEL classification numbers: E21, E30, O10, O41

${ }^{*}$ We appreciate financial supports from the Joint Usage/Research Center Project of ISER from the Ministry of Education, Culture, Sports, Science and Technology. All remaining errors are ours.

Corresponding author: College of Economics, Aoyamagakuin University, 4-4-25, shibuya, shibuya-ku, Tokyo, 150-8366, Japan. E-mail: t-hori@ aoyamagakuin.jp.

Institute of Social and Economic Research, Osaka University, 6-1 Mihogaoka, Ibaraki, Osaka 567-0047 Japan, e-mail: ikefuji@iser.osaka-u.ac.jp

${ }^{\S}$ Institute of Economic Research, Kyoto University, Yoshida Honmachi, Sakyo-ku, Kyoto, 606-8501 Japan, e-mail: mino@kier.kyoto-u.ac.jp 


\section{Introduction}

Seeking to answer the question of why there is so much evidence of large disparities in structural change and economic growth across countries is one of the central concerns of growth economics. The existing literature on economic growth and development provides some important insight into what factors drive structural change. Nevertheless, the previous models are not completely consistent with observations of varying structural change as discussed by Buera and Kaboski (2009). ${ }^{1}$ In this paper, we show that commodity-specific consumption externalities can be a source of the diverse patterns of structural change using a simple two-sector general equilibrium model.

Broadly speaking, two approaches have been used to study structural change. The one is based on the assumption for production technology, while the other relies on nonhomothetic preferences assumption. In the studies concerning technological explanations, following Baumol's (1967) seminal work, Ngai and Pissarides (2007) assume that the growth rates of total factor productivity (TFP) are different across sectors so as to derive supply-induced structural change. They demonstrate that an aggregate balanced growth path exists only if the utility function is logarithmic in the consumption composite consisting of one manufacturing good and an arbitrary number of consumption goods. Acemoglu and Guerrieri (2008) show that differences in factor proportions combined with capital deepening lead to an economy that features nonbalanced growth at the sectoral level, and that aggregate growth is consistent with the well-known Kaldor facts suggesting approximate constancy of aggregate growth. In a framework based on demand-side reasons, Kongsamut et al. (2001) employ a Stone-Geary utility function to illustrate the dynamic of sectoral labor reallocation. However, in order for their growth model to be reconciled with the Kaldor facts, a knife-edge condition is required. ${ }^{2}$ In contrast, Foellmi and Zwimüller (2008) introduce a hierarchic utility function to obtain non-linear Engel-curves for the various products, which generates consumption cycles. Provided that demand functions only depend on the relative position of the product in the hierarchy, they also show that structural change is consistent with the Kaldor facts. ${ }^{3}$

Unlike most previous works, we assume neither non-homothetic preferences of individuals nor sectoral differences in productivity growth. In fact, we assume that consumers' preferences are homothetic in their private consumption and that each sector employs AK technology of which TFP is constant. However, if commodity-specific consumption externalities are introduced, the utility function exhibits non-homotheticity from the social perspective, which generates structural change reconciled with Kaldor facts. Under the as-

\footnotetext{
${ }^{1}$ They find that traditional explanations are (1) unable to account for observed sectoral movement; (2) reliant on an unrealistic elasticity of substitution; and (3) inconsistent with the data between trends in sectoral labor shares and trends in sectoral output shares.

${ }^{2}$ The restriction requires that the market value of subsistence consumption of agricultural goods and that of home production of services are the same.

${ }^{3}$ In the case of an endogenous R\&D framework, they show that hierarchic preferences stimulates the incentive to innovate, which may generate multiple equilibria. Recently, Guilló et al. (2011) construct a multisector overlapping generations model of endogenous technical change with the aim of showing that biased TFP growth and labor movement across sectors can be an endogenous response to the non-homothetic preference.
} 
sumption that the elasticity of substitution between the two goods is not equal unity, we show that the economy converges to a balanced growth equilibrium when the same degree of consumption externalities is applied commonly to both types of goods. However, when the degrees of consumption externalities are different from each other, the output of one sector grows faster than the other sector, whereas the aggregate economy exhibits balanced growth. Moreover, we illustrate that the pattern of structural change depends on the initial levels of benchmark consumption for given the initial capital stock in the latter case. Thus, commodity-specific consumption externalities can drive structural change without sectorbiased technical change and non-homotheticity of private preferences. In addition, there may exist various patterns in structural change.

The role of commodity-specific consumption externalities has been examined by some authors such as Ravn et al. (2006), Doi and Mino (2008) and Hori (2011) who assume that consumers put the same importance on all types of consumption of other households. In such a situation, a consumer would care equally about consumption of bread and car of other consumers. However, the recent studies of the behavioral economics provide the evidence that consumers put the different importance on the different types of consumption of other consumers. ${ }^{4}$ Our model generalizes to allow for differing degrees of consumption externalities between different goods.

The rest of the paper is organized as follows: The next section sets up the model. Section 3 examines the evolution of the aggregate economy and shows the aggregate economy reconcile with Kaldor facts. Section 4 examines the dynamics of the two sectors and describes an equilibrium. Section 5 analyzes the dynamic system for the case that the degrees of consumption externalities of two goods are same and shows that structural change does not occur. Section 6 analyzes the dynamic system and the stability of the equilibrium when the degrees of consumption externalities of two goods are different and describes how structural change occurs. Concluding remarks are given in Section 7.

\section{The Model}

\subsection{Firms}

Time is continuous and denoted by $t \in[0, \infty)$. We consider a competitive economy that has two sectors. Sector 1 produces a good (called good 1), which can be either invested for capital accumulation or consumed. Sector 2 supplies a pure consumption good (called good 2). ${ }^{5}$ Good 1 is chosen as numeraire. The price of good 2 at time $t$ is denoted by $p_{t}$.

Firms in each sector are homogeneous. The number of firms in each sector is normalized to one. The representative firm in Sector $i(=1,2)$ produces good $i$ by using the following

\footnotetext{
${ }^{4}$ See Solnick and Hemenway (1998), Alpizar et al. (2005), and Carlsson et al. (2007), for example. They investigate the positionality degree for different goods with empirical or experimental approaches.

${ }^{5}$ Ngai and Pissarides (2007) consider the economy with $m$ sectors where one of the $m$ sectors produces capital stock as well as consumption good, and the remaining $m-1$ sectors produce only consumption goods. The production side of our model is a special case of Ngai and Pissarides (2007) where $m=2$ holds.
} 
technology:

$$
Y_{i, t}=A_{i} K_{i, t}^{\alpha}\left(B_{i, t} l_{i, t}\right)^{1-\alpha}, \quad 0<\alpha<1,
$$

where $Y_{i, t}, K_{i, t}, l_{i, t}$ and $A_{i}>0$ are the output level, the capital input, the labor input and the productivity in Sector $i$, respectively. It is assumed that the two sectors have the same capital intensity. $B_{i, t}$ represents the sector-specific externalities. Let us denote the average capital and labor inputs of Sector $i(=1,2)$ as $\bar{K}_{i, t}$ and $\bar{l}_{i, t}$, respectively. We have $K_{i, t}=\bar{K}_{i, t}$ and $l_{i, t}=\bar{l}_{i, t}$ in equilibrium. We specify $B_{i, t} \equiv \bar{K}_{i, t} / \bar{l}_{i, t}$ so that the total production function of the two sectors take simple $A K$-forms, $Y_{i, t}=A_{i} K_{i, t}$, as in Carrol et al. $(1997,2000)$ who study the role of consumption externalities in a one-sector growth model. The simplification allows us to focus on the role of consumption externalities.

We assume that capital depreciation rate is equal to zero. Profit maximization in both sectors yields:

$$
\begin{gathered}
r_{t}=\alpha A_{1}=p_{t} \alpha A_{2} \quad \text { and } \quad p_{t}=\frac{A_{1}}{A_{2}}, \\
w_{t}=(1-\alpha) A_{1} \frac{K_{1, t}}{l_{1, t}}=p_{t}(1-\alpha) A_{2} \frac{K_{2, t}}{l_{2, t}} .
\end{gathered}
$$

The first line shows that due to our specification of the technology, the rental rate of capital, $r$, and the price of good 2, $p$, are both constant over time, which allows us to focus on the role of consumption externalities. In the following discussion, we omit the index $t$ from $r$ and $p$. The second line gives the wage rate. The capital and labor market equilibrium conditions are:

$$
\begin{aligned}
K_{1, t}+K_{2, t} & =K_{t}, \\
l_{1, t}+l_{2, t} & =l_{t},
\end{aligned}
$$

where $K_{t}$ and $l_{t}$ is the aggregate capital stock and labor supply. There is free capital and labor mobility between the two sectors. The aggregate production of the economy is given by:

$$
Y_{t} \equiv Y_{1, t}+p Y_{2, t}=A_{1} K_{t}
$$

\subsection{Households}

There is a continuum of identical households whose number is normalized to one. The representative household consumes goods 1 and 2 . The household inelastically supplies one unit of labor, $l_{t}=1$. We assume that the instantaneous utility of the representative household depends not only on her own consumption of the two goods but also on the benchmark levels of consumption that are determined through outward-looking habit formation. The instantaneous subutility of the representative household at time $t$ is given by:

$$
u_{t}= \begin{cases}{\left[\gamma\left(c_{1, t} h_{1, t}{ }^{-\theta_{1}}\right)^{\frac{\varepsilon-1}{\varepsilon}}+(1-\gamma)\left(c_{2, t} h_{2, t}{ }^{-\theta_{2}}\right)^{\frac{\varepsilon-1}{\varepsilon}}\right]^{\frac{\varepsilon}{\varepsilon-1}},} & \text { if } \varepsilon \neq 1, \\ \left(c_{1, t} h_{1, t}{ }^{-\theta_{1}}\right)^{\gamma}\left(c_{2, t} h_{2, t}{ }^{-\theta_{2}}\right)^{1-\gamma}, & \text { if } \varepsilon=1 .\end{cases}
$$


where $c_{i, t}$ is consumption of good $i(=1,2)$ at time $t, \varepsilon>0$ is the elasticity of substitution between the two goods, and $\gamma \in(0,1)$ represents the importance of good 1. Here, $h_{i, t}$ denotes the benchmark level of consumption of good $i$ at time $t$. The presence of $h_{i, t}$ in (4) represents the commodity-specific consumption externalities, as in Ravn et al. (2006), Doi and Mino (2008) and Hori (2011). As we discuss later, $\theta_{i} \in[0,1)$ is associated with commodity-specific consumption externalities. We assume that $h_{i, t}$ is a weighted sum of the past average consumption of good $i$ up to the time $t$ :

$$
h_{i, t}=\phi_{i} \int_{-\infty}^{t} e^{-\phi_{i}(t-u)} \bar{c}_{i, u} d u, \quad \phi_{i}>0,
$$

where $\bar{c}_{i, u}$ is the average consumption of good $i$ at time $u$ in the economy at large. Differentiating the both sides of the above equation with respect to $t$ yields:

$$
\dot{h}_{i, t}=\phi_{i, t}\left(\bar{c}_{i, t}-h_{i, t}\right) \text {. }
$$

Concerning (4), the three points should be mentioned. First, when $\varepsilon \neq 1$ holds, $u_{t}$ can be written as:

$$
u_{t}=\left\{\gamma\left[c_{1, t}{ }^{1-\theta_{1}}\left(\frac{c_{1, t}}{h_{1, t}}\right)^{\theta_{1}}\right]^{\frac{\varepsilon-1}{\varepsilon}}+(1-\gamma)\left[c_{2, t}{ }^{1-\theta_{2}}\left(\frac{c_{2, t}}{h_{2, t}}\right)^{\theta_{2}}\right]^{\frac{\varepsilon-1}{\varepsilon}}\right\}^{\frac{\varepsilon}{\varepsilon-1}}
$$

The subutility depends on the relative level of consumption, $c_{i} / h_{i}$, as well as the absolute level of consumption, $c_{i}{ }^{6}$ When both $\theta_{1}$ and $\theta_{2}$ are equal to zero, only the absolute levels of consumption matter. If $\theta_{1}=\theta_{2}=1$, only the relative level of consumption is all that matters. For other values of $\theta_{i}(\in(0,1))$, both are important. As in Carroll et al. (1997, 2000), we assume that $0 \leq \theta_{i}<1$. When $\theta_{i}$ is strictly positive, an increase in $h_{i, t}$ negatively affects $u_{t}$. The preference of each household exhibits jealousy toward consumption of others. Because the relative consumption of good $i$ becomes more important as $\theta_{i}$ increases, we interpret $\theta_{i}$ as an indicator of how much each household cares about the relative consumption of good $i$. We call $\theta_{i}$ the degree of consumption externalities of good $i$. It is also to be noted that if $\phi_{i}=+\infty$, then $h_{i, t}=\bar{c}_{i, t}$ holds so that external effects are only intratemporal.

The second relevant point is the degrees of consumption externalities. As discussed in Introduction, the previous studies on the commodity-specific consumption externalities assume that consumers put the same importance on the relative consumption of all good, which means $\theta_{1}=\theta_{2}$. However, the recent studies of the behavioral and experimental economics suggest that the importance of the relative consumption varies depending on characteristics of goods. Therefore, we consider the case of $\theta_{1} \neq \theta_{2}$ as well as that of $\theta_{1}=\theta_{2}$. Moreover, these studies typically find that people tend to care more about the relative consumption of the more visible goods like cars than that of the less visible good. In our setting, we can reasonably consider good 1 that is used as capital good to be the more visible good. So in what follow, the case of $\theta_{1}>\theta_{2}$ is more relevant, although we also pay attention to the case of $\theta_{1} \leq \theta_{2}$.

\footnotetext{
${ }^{6}$ When $\varepsilon=1$ holds, $u_{t}$ can be written as $u_{t}=\left\{c_{1, t}^{1-\theta_{1}}\left(c_{1, t} / h_{1, t}\right)^{\theta_{1}}\right\}^{\gamma}\left\{c_{2, t}^{1-\theta_{2}}\left(c_{2, t} / h_{2, t}\right)^{\theta_{2}}\right\}^{1-\gamma}$. Again, $u_{t}$ depends on the relative level of consumption, $c_{i} / h_{i}$, as well as the absolute level of consumption, $c_{i}$.
} 
The final point is concerned with homotheticity. Some authors such as Kongsamut et al. (2001) employ a non-homothetic utility function in order to construct a growth model where outputs of different sectors grow at different rates. Apparently, (4) is homothetic from the perspective of private consumption, $c_{1, t}$ and $c_{2, t}$. In the following discussion, we will observe that even under a homothetic utility function, the presence of commodity-specific consumption externalities gives rise to nonbalnaced growth.

The utility of the representative household is given by:

$$
U=\int_{0}^{\infty} e^{-\rho t} \ln u_{t} d t
$$

where $u_{t}$ is given by (4) and $\rho>0$ is the subjective discount rate. To ensure a positive growth rate in equilibrium, we assume $\alpha A_{1}>\rho .^{7}$ We use a logarithmic utility in (6) because Ngai and Pissarides (2007) show that if the instantaneous utility function is a CES function, structural change is consistent with balanced growth of the aggregate economy only when the intertemporal elasticity of substitution is equal to one. The budget constraint is:

$$
\begin{aligned}
\dot{a}_{t} & =r a_{t}+w_{t}-E_{t}, \\
E_{t} & =c_{1, t}+p c_{2, t},
\end{aligned}
$$

where $a_{t}$ denotes the asset holdings of the household at time $t$ that is equal to $K_{t}$ in equilibrium. We solve the optimization problem of the household in two steps. In the first step, $u_{t}$ is maximized subject to (7b), which yields:

$$
\frac{c_{2, t}}{c_{1, t}}=\left(\frac{1-\gamma}{\gamma p}\right)^{\varepsilon}\left(\frac{h_{2, t}^{\theta_{2}}}{h_{1, t}^{\theta_{1}}}\right)^{1-\varepsilon} .
$$

Given $h_{1, t}$ and $h_{2, t},(8)$ shows that the relative demand, $c_{2, t} / c_{1, t}$, is a decreasing function of the relative price, $p$. Assume $\theta_{1}>0$ and $\theta_{2}>0$. Consider the case where the elasticity of substitution is large so that $\varepsilon>1$ holds. An increase in $h_{1, t}\left(h_{2, t}\right)$ reduces the relative demand for $c_{1, t}\left(c_{2, t}\right)$. Therefore, in our specification, the preference of the household exhibits "running away from the Joneses (RAJ)" when $\varepsilon>1$. When $\varepsilon<1$ holds, an increase in $h_{1, t}\left(h_{2, t}\right)$ has a positive effect on the relative demand for $c_{1, t}\left(c_{2, t}\right)$. Then, "catching up with the Joneses (CUJ)" prevails. Hence, a smaller $\varepsilon$ represents a stronger degree of conformity among consumers. The intuition is as follows: As $h_{1, t}$ increases, consumption of good 1 relative to its benchmark, $c_{1, t} / h_{1, t}$, decreases, which has negative effects on $u_{t}$. When the elasticity of substitution is large, the household attempts to compensate the negative effects of $h_{1, t}$ by substituting consumption of good 1 with that of good 2. Then, RAJ prevails. When the elasticity of substitution is small, the household attempts to compensate the negative effects of $h_{1, t}$ by consuming good 1 more. Then, her preference exhibits CUJ. Most of the existing studies in the literature assume CUJ, rather than RAJ. The case of $\varepsilon<1$ is more related to the literature although we also pay attention to the case of $\varepsilon>1$.

From (7b) and (8), the following equations are derived:

$$
c_{1, t}=\frac{E_{t}}{1+p z\left(\frac{h_{2, t}^{\theta_{2}}}{h_{1, t}{ }^{\theta_{1}}}\right)^{1-\varepsilon}} \equiv \chi_{t} E_{t} \text {, and } p c_{2, t}=\left(1-\chi_{t}\right) E_{t} \text {. }
$$

\footnotetext{
${ }^{7}$ As we will see later, the growth rate of the economy is given by $\alpha A_{1}-\rho$ in a long-run equilibrium.
} 
where $z \equiv\left(\frac{1-\gamma}{\gamma p}\right)^{\varepsilon}$. Then, (4) can be written as:

$$
\left.u_{t}=E_{t}\left[\gamma\left(\chi_{t} h_{1, t}{ }^{-\theta_{1}}\right)^{\frac{\varepsilon-1}{\varepsilon}}+(1-\gamma)\left(\frac{1-\chi_{t}}{p} h_{2, t}\right)^{-\theta_{2}}\right)^{\frac{\varepsilon-1}{\varepsilon}}\right]^{\frac{\varepsilon}{\varepsilon-1}} .
$$

The second step of the household's problem is to maximize (6) subject to (7a) and the above equation. We then obtain:

$$
\dot{E}_{t}=(r-\rho) E_{t} .
$$

The transversality condition (TVC) is given by $\lim _{t \rightarrow \infty} a_{t} e^{-r t}=0$. Since $a_{t}=K_{t}$ holds in equilibrium, TVC is satisfied if $\lim _{t \rightarrow \infty} \dot{K}_{t} / K_{t}<r\left(=\alpha A_{1}\right)$.

Before closing this subsection, we derive the next two equations for later use.

$$
\begin{aligned}
& \frac{\dot{c}_{1, t}}{c_{1, t}}-\frac{\dot{c}_{2, t}}{c_{2, t}}=(1-\varepsilon)\left(\theta_{1} \frac{\dot{h}_{1, t}}{h_{1, t}}-\theta_{2} \frac{\dot{h}_{2, t}}{h_{2, t}}\right), \\
& \frac{\dot{c}_{1, t}}{c_{1, t}}=\alpha A_{1}-\rho+(1-\varepsilon)\left(1-\frac{c_{1, t}}{E_{t}}\right)\left(\theta_{1} \frac{\dot{h}_{1, t}}{h_{1, t}}-\theta_{2} \frac{\dot{h}_{2, t}}{h_{2, t}}\right) .
\end{aligned}
$$

We obtain the first equation by differentiating both sides of (8) with respect to $t$. The derivation of (12) is presented in Appendix A. Equation (12) shows that when $\theta_{1}>0$ and $\theta_{2}$ hold, $\dot{h}_{1, t} / h_{1, t}$ has a positive (negative) effect on $\dot{c}_{1, t} / c_{1, t}$ if $\varepsilon<(>) 1$ holds while $\dot{h}_{2, t} / h_{2, t}$ has a negative (positive) effect on $\dot{c}_{1, t} / c_{1, t}$. This is because when $\varepsilon<(>) 1$ holds, CUJ (RAJ) prevails.

\subsection{Goods Market}

Good 1 can be either invested for capital accumulation or consumed and good 2 is a pure consumption good. Thus the goods market clearing conditions are:

$$
\begin{aligned}
\dot{K}_{t} & =A_{1} K_{1, t}-c_{1, t}, \\
c_{2, t} & =A_{2} K_{2, t} .
\end{aligned}
$$

The above two equations, together with (1a) and (7b), imply that the aggregate capital evolves according to:

$$
\dot{K}_{t}=A_{1} K_{t}-E_{t}
$$

\section{The Aggregate Economy}

Before examining the dynamics of the two sectors, we examine the evolution of the aggregate variables, $K_{t}$ and $E_{t}$. Define $\xi_{t} \equiv E_{t} / K_{t}$. From (10) and (13), we obtain:

$$
\dot{\xi}_{t}=\left\{\xi_{t}-\left[(1-\alpha) A_{1}+\rho\right]\right\} \xi_{t} .
$$

Figure 1 shows the graph of the dynamics of $\xi$. The path that $\xi_{t}$ converges to zero cannot be equilibrium because $\dot{K}_{t} / K_{t}$ converges to $A_{1}$ and TVC is not satisfied. The path that $\xi_{t}$ tends 
to $+\infty$ is not equilibrium either because $K_{t}$ converges to zero. Hence, the economy always stay at $\xi^{*} \equiv(1-\alpha) A_{1}+\rho$ and then we have:

$$
E_{t}=\left[(1-\alpha) A_{1}+\rho\right] K_{t}
$$

for all $t \geq 0$. In equilibrium, $K_{t}$ and $E_{t}$ grow at the same constant rate, $g^{*} \equiv \alpha A_{1}-\rho$. The capital income share remains constant at $\alpha=r K_{t} / Y_{t}$. The interest rate also remains constant. We then obtain the next proposition.

\section{Proposition 1}

In equilibrium, $\xi_{t}$ remains constant at $\xi^{*} \equiv(1-\alpha) A_{1}+\rho$ over time. $K_{t}$ and $E_{t}$ grow at $g^{*} \equiv \alpha A_{1}-\rho$. The capital income share and the interest rate remain constant.

Proposition 1 shows that the aggregate economy satisfies the facts pointed out by Kaldor. Several points should be mentioned. Because of our specification of the technology, the interest rate and the growth rate of $K_{t}$ always remain constant. If there are no sector-specific externalities and then the production function takes a standard Cobb-Douglas form, the transitional dynamics arise and hence the interest rate and the growth rate of $K_{t}$ no longer remain constant over time. Note that the studies about structural change, including Acemoglu and Guerrieri (2008) and Kongsamut (2001), also focus on the steady state equilibrium where the interest rate remains constant and capital stock grows at a constant rate. Therefore, our analysis is in line with the literature. Second, as shown in Carrol et al. (1997, 2000), when the benchmark level of consumption depends on the past consumption, the transitional dynamics arises even if the production technology takes an $A K$ form. Because of the logarithmic utility, the dynamics of the aggregate economy is independent of the benchmark level of consumption and hence no transitional dynamics arises in our model. Finally, the fact that $K_{t}$ and $E_{t}$ grows at the same constant rate does not necessarily mean that the two sectors also grow at the same rate and there are no transitional dynamics of each production sector. In fact, the subsequent sections show that under reasonable conditions, the two sectors grow at different rates and hence structural change occurs. Because consumption externalities have no influence on the aggregate economy as mentioned just above, we can examine the direct consequence of consumption externalities on structural change.

[Figure 1]

\section{Dynamic System of the Two Sectors}

To examine the dynamics of the two sectors, we define $x_{1, t} \equiv c_{1, t} / K_{t}, x_{2, t} \equiv p c_{2, t} / K_{t}$ and $\eta_{i, t} \equiv h_{i, t} / K_{t}(i=1,2)$.

From (7b) and (14), we have:

$$
x_{1, t}+x_{2, t}=(1-\alpha) A_{1}+\rho .
$$


If we use the definitions of $x_{i, t}$ and $\eta_{i, t}$ and the fact that $\bar{c}_{i, t}=c_{i, t}$ holds in equilibrium, we can derive the following three differential equations from (5), (12) and (14):

$$
\begin{aligned}
& \dot{x}_{1, t}=(1-\varepsilon)\left(1-\frac{x_{1, t}}{(1-\alpha) A_{1}+\rho}\right)\left[\theta_{1} \phi_{1}\left(\frac{x_{t}}{\eta_{1, t}}-1\right)-\theta_{2} \phi_{2}\left(\frac{x_{2, t}}{p \eta_{2, t}}-1\right)\right] x_{t}, \\
& \dot{\eta}_{1, t}=\phi_{1} x_{t}-\left(\phi_{1}+g^{*}\right) \eta_{1, t}, \\
& \dot{\eta}_{2, t}=\frac{\phi_{2}}{p} x_{2, t}-\left(\phi_{2}+g^{*}\right) \eta_{2, t},
\end{aligned}
$$

where $x_{2, t}=(1-\alpha) A_{1}+\rho-x_{1, t}$ and $0 \leq x_{1, t} \leq(1-\alpha) A_{1}+\rho . \eta_{1, t}$ and $\eta_{2, t}$ are state variables. From the first equation of (9) and (14), we can derive:

$$
x_{1, t}=\frac{(1-\alpha) A_{1}+\rho}{1+p z\left(\frac{h_{2, t} \theta_{2}}{h_{1, t}{ }^{\theta_{1}}}\right)^{1-\varepsilon}} .
$$

This shows that the initial value of $x_{1, t}$ is determined by $h_{1,0}$ and $h_{2,0}$. Hence, we should treat $x_{1,0}$ as a predetermined variable. Given $x_{1,0}, \eta_{1,0}$ and $\eta_{2,0}$, when the degrees of consumption externalities are different between goods 1 and $2\left(\theta_{1} \neq \theta_{2}\right)$, the dynamic system consists of (16a)-(16c).

When the degree of consumption externalities of good 1 is the same as that of good 2 $\left(\theta_{1}=\theta_{2} \equiv \theta(0 \leq \theta<1)\right), x_{1, t}$ becomes a function of $\eta_{1, t}$ and $\eta_{2, t}$ from (17).

$$
x_{1, t}=\frac{(1-\alpha) A_{1}+\rho}{1+p z\left(\frac{\eta_{2, t}}{\eta_{1, t}}\right)^{\theta(1-\varepsilon)}} .
$$

Substituting (17) into (16b) and (16c) using (15), we can derive the dynamic system of $\eta_{1, t}$ and $\eta_{2, t}$ given $\eta_{1,0}$ and $\eta_{2,0}$.

We next define a stationary equilibrium.

\section{Definition}

A stationary equilibrium (SE) is an equilibrium where $\dot{x}_{1, t}=\dot{\eta}_{1, t}=\dot{\eta}_{2, t}=0$ holds and $c_{1, t}$, $c_{2, t}, h_{1, t}$ and $h_{2, t}$ grow at constant rates.

It should be noted that in an SE, $c_{1, t}, c_{2, t}, h_{1, t}$ and $h_{2, t}$ do not necessarily grow at the same rate. An SE requires only that the grow rates of $c_{1, t}, c_{2, t}, h_{1, t}$ and $h_{2, t}$ are constant over time. In the following discussion, we omit time index $t$ from variables that are constant over time when we analyze an SE.

When $\varepsilon=1$ holds, the benchmark levels of consumption, $h_{1, t}$ and $h_{2, t}$, do not affect the demand for both goods 1 and 2 (see (8)). Consequently, consumption externalities have no influence on $x_{1, t}$ in equilibrium (see (16a), (17) and (18)). Because we are interested in the effects of consumption externalities, we assume $\varepsilon \neq 1$ in the following discussion. Before closing this section, we prove the next proposition concerning the existence of SE.

\section{Proposition 2}

Suppose $\varepsilon \neq 1$ and $\theta_{1} \neq \theta_{2}$. If there exists an $S E, \dot{c}_{1, t} / c_{1, t} \neq \dot{c}_{2, t} / c_{2, t}$ must hold in the SE. 
(Proof) Suppose that there exists an SE where $\dot{c}_{1, t} / c_{1, t}=\dot{c}_{2, t} / c_{2, t}$ holds. Then, (11) implies $\theta_{1} \dot{h}_{1, t} / h_{1, t}=\theta_{2} \dot{h}_{2, t} / h_{2, t}$ because of $\varepsilon \neq 1$. When $\dot{c}_{1, t} / c_{1, t}=\dot{c}_{2, t} / c_{2, t}$ holds, $\dot{c}_{1, t} / c_{1, t}=\dot{c}_{2, t} / c_{2, t}=$ $g^{*}\left(\equiv \dot{E}_{t} / E_{t}\right)$ must hold so that (7b) is satisfied. Because $c_{1, t}$ and $c_{2, t}$ grows at the same rate as $K_{t}, x_{1, t}$ and $x_{2, t}$ become constant. We denote those constants as $\hat{x}_{1}$ and $\hat{x}_{2}$. From (16b) and (16c), we know that $\eta_{1, t}$ and $\eta_{2, t}$ converge to $\phi_{1} \hat{x}_{1} /\left(\phi_{1}+g^{*}\right)$ and $\phi_{2} \hat{x}_{2} /\left\{p\left(\phi_{2}+g^{*}\right)\right\}$, respectively. By definition of $\eta_{1, t}$ and $\eta_{2, t}$, we must have $\lim _{t \rightarrow+\infty} \dot{h}_{1, t} / h_{1, t}=g^{*}=\lim _{t \rightarrow+\infty} \dot{h}_{1, t} / h_{1, t}$, which contradicts $\theta_{1} \dot{h}_{1, t} / h_{1, t}=\theta_{2} \dot{h}_{2, t} / h_{2, t}$ because of $\theta_{1} \neq \theta_{2}$. Then, $\dot{c}_{1, t} / c_{1, t} \neq \dot{c}_{2, t} / c_{2, t}$ must hold in the SE.

Proposition 2 tells that when $\theta_{1} \neq \theta_{2}$ holds, there is possibility that the two sectors grow at different rates and hence structural change occurs. In the following discussion, we first consider the case of $\theta_{1}=\theta_{2}$ and show that structural change does not occur. After that, we proceed to the case of $\theta_{1} \neq \theta_{2}$ and observe that the presence of consumption externalities is a source of structural change.

\section{The Case of $\theta_{1}=\theta_{2} \equiv \theta(0 \leq \theta<1)$}

When $\theta_{1}=\theta_{2} \equiv \theta(0 \leq \theta<1)$ holds, the dynamic system is given by (16b), (16c) and (18). We first derive an SE. By setting $\dot{\eta}_{1, t}=\dot{\eta}_{2, t}=0$ in (16b) and (16c), we obtain:

$$
\phi_{1} x_{1}=\left(\phi_{1}+g^{*}\right) \eta_{1} \text { and } \phi_{2} x_{2}=p\left(\phi_{2}+g^{*}\right) \eta_{2} .
$$

After we divide both sides of the first equation by those of the second one and rearrange the resulting equation using (15) and (18), we obtain:

$$
\frac{\eta_{2}}{\eta_{1}}=\left[\frac{\phi_{2}\left(\phi_{1}+g^{*}\right) z}{\phi_{1}\left(\phi_{2}+g^{*}\right)}\right]^{\frac{1}{1+\theta(\varepsilon-1)}} \equiv \Delta .
$$

By use of (15), (18) and the above equation, we rearrange the two equations of (19) so as to yield the SE values of $\eta_{1}$ and $\eta_{2}$ :

$$
\eta_{1}^{*} \equiv \frac{(1-\alpha) A_{1}+\rho}{\phi_{1}+g^{*}} \frac{\phi_{1}}{1+p z \Delta^{\theta(1-\varepsilon)}}, \quad \text { and } \quad \eta_{2}^{*} \equiv \frac{(1-\alpha) A_{1}+\rho}{\phi_{2}+g^{*}} \frac{\phi_{2} z \Delta^{\theta(1-\varepsilon)}}{1+p z \Delta^{\theta(1-\varepsilon)}} .
$$

Because $\eta_{i}$ remains constant at $\eta_{i}^{*}$ that is strictly larger than zero, we have $\dot{h}_{1, t} / h_{1, t}=$ $\dot{h}_{2, t} / h_{2, t}=g^{*}$. From (18), $x_{1, t}$ also becomes constant and strictly positive. Hence, we find that $\dot{c}_{1, t} / c_{1, t}=\dot{c}_{2, t} / c_{2, t}=g^{*}$. Structural change does not occur in the SE we have just derived. Note that these results hold regardless of whether $\theta$ is equal to zero or not.

We next examine the transitional dynamics with the help of phase diagrams. From (18) and the first equation of (19), we can derive $\dot{\eta}_{1}=0$ locus:

$$
\eta_{2}=(p z)^{\frac{1}{\theta(\varepsilon-1)}}\left[\frac{\phi_{1}\left\{(1-\alpha) A_{1}+\rho\right\}}{\phi_{1}+g^{*}}-\eta_{1}\right]^{\frac{1}{\theta(1-\varepsilon)}} \eta_{1}^{\frac{1+\theta(\varepsilon-1)}{\theta(\varepsilon-1)}} .
$$

Note that we have $1+\theta(\varepsilon-1)>1-\theta>0$ because of $\theta \in[0,1)$ and $\varepsilon>0(\varepsilon \neq 1)$. As shown Panel (a) of Figure 2, when $\varepsilon<1$ holds, $\eta_{2}$ tends to $+\infty$ as $\eta_{1}$ approaches zero while 
$\eta_{2}$ tends to zero as $\eta_{1}$ approaches $\phi_{1}\left[(1-\alpha) A_{1}+\rho\right] /\left(\phi_{1}+g^{*}\right)$. In the region above (below) $\dot{\eta}_{1}=0$ locus, we have $\dot{\eta}_{1}<(>) 0$. When $\varepsilon>1$ holds, $\eta_{2}$ tends to zero as $\eta_{1}$ approaches zero while $\eta_{2}$ tends to $+\infty$ as $\eta_{1}$ approaches $\phi_{1}\left[(1-\alpha) A_{1}+\rho\right] /\left(\phi_{1}+g^{*}\right)$. In the region above (below) $\dot{\eta}_{1}=0$ locus, we have $\dot{\eta}_{1}>(<) 0$. From (15), (18) and the second equation of (19), we can derive $\dot{\eta}_{2}=0$ locus:

$$
\eta_{1}=(p z)^{\frac{1}{\theta(1-\varepsilon)}}\left[\frac{\phi_{2}\left\{(1-\alpha) A_{1}+\rho\right\}}{p\left(\phi_{2}+g^{*}\right)}-\eta_{2}\right]^{\frac{1}{\theta(1-\varepsilon)}} \eta_{2}^{\frac{1+\theta(\varepsilon-1)}{\theta(\varepsilon-1)}} .
$$

As shown in Panel (a) of Figure 2, when $\varepsilon<1$ holds, $\eta_{1}$ tends to $+\infty$ as $\eta_{2}$ approaches zero while $\eta_{1}$ tends to zero as $\eta_{2}$ approaches $\phi_{2}\left[(1-\alpha) A_{1}+\rho\right] /\left[p\left(\phi_{2}+g^{*}\right)\right]$. In the region above (below) $\dot{\eta}_{2}=0$ locus, we see that $\dot{\eta}_{2}<(>) 0$. When $\varepsilon>1$ holds, $\eta_{1}$ tends to zero as $\eta_{2}$ approaches zero while $\eta_{1}$ tends to $+\infty$ as $\eta_{2}$ approaches $\phi_{2}\left[(1-\alpha) A_{1}+\rho\right] /\left[p\left(\phi_{2}+g^{*}\right)\right]$. In the region above (below) $\dot{\eta}_{2}=0$ locus, we have $\dot{\eta}_{2}<(>) 0$. Phase diagrams in Figure 2 show that the SE is stable and the economy converges to the SE.

[Figure 2]

We then obtain the following proposition.

\section{Proposition 3}

Suppose $\varepsilon \neq 1$ and $\theta_{1}=\theta_{2}=\theta \geq 0$. The economy converges to the SE where $\dot{c}_{1, t} / c_{1, t}=$ $\dot{c}_{2, t} / c_{2, t}=\dot{h}_{1, t} / h_{1, t}=\dot{h}_{2, t} / h_{2, t}=g^{*}$ holds.

Proposition 3 holds regardless of whether $\theta$ is equal to zero or strictly positive. When $\theta_{1}=$ $\theta_{2}$ holds, regardless of the presence of consumption externalities, growth is asymptotically balanced in the sense that the two sectors grow at the same asymptotic rate.

The contraposition of Proposition 2 tells that if there exists an SE where $\dot{c}_{1, t} / c_{1, t}=$ $\dot{c}_{2, t} / c_{2, t}$ holds, $\theta_{1}=\theta_{2}$ holds in case of $\varepsilon \neq 1$. We obtain the next corollary from Propositions 2 and the results of this section.

\section{Corollary}

Suppose $\varepsilon \neq 1$. There exist an SE where $\dot{c}_{1, t} / c_{1, t}=\dot{c}_{2, t} / c_{2, t}$ if and only if $\theta_{1}=\theta_{2}=\theta$ $(0 \leq \theta<1)$ holds.

The next section shows that when the degrees of consumption externalities are different between different goods $\left(\theta_{1} \neq \theta_{2}\right)$, the presence of consumption externalities generates structural change.

\section{The Case of $\theta_{1} \neq \theta_{2}$}

Again, let us assume that $\varepsilon \neq 1$. When $\theta_{1} \neq \theta_{2}$ holds, the dynamic system is given by (16a)-(16c). In this case, there is no SE where $c_{1, t}$ and $c_{2, t}$ grow at the same rate as shown in Proposition 2. However, the next proposition shows the existence of an SE where the two sectors grow at the different (asymptotic) rates. 


\section{Proposition 4}

(i) Suppose $(1-\varepsilon)\left(\theta_{1}-\theta_{2}\right)>0$. There exists an SE where the followings hold:

$$
\begin{aligned}
& \frac{\dot{c}_{1, t}}{c_{1, t}}=\frac{\dot{h}_{1, t}}{h_{1, t}}=\alpha A_{1}-\rho \equiv g^{*}, \\
& \frac{\dot{c}_{2, t}}{c_{2, t}}=\frac{\dot{h}_{2, t}}{h_{2, t}}=\frac{\dot{K}_{2, t}}{K_{2, t}}=\frac{1+(\varepsilon-1) \theta_{1}}{1+(\varepsilon-1) \theta_{2}} g^{*} \equiv \hat{g}^{*}\left(0<\hat{g}^{*}<g^{*}\right), \\
& x_{1}=(1-\alpha) A_{1}+\rho\left(\equiv \hat{x}^{*}\right), \eta_{1}=\frac{\phi_{1}\left[(1-\alpha) A_{1}+\rho\right]}{\phi_{1}+g^{*}}\left(\equiv \hat{\eta}_{1}^{*}\right), \eta_{2}=0\left(\equiv \hat{\eta}_{2}^{*}\right), \\
& \lim _{t \rightarrow \infty} \frac{c_{2, t}}{h_{2, t}}=\lim _{t \rightarrow \infty} \frac{A_{2} k_{2, t}}{\eta_{2, t}}=\frac{\phi_{2}+\hat{g}^{*}}{\phi_{2}} .
\end{aligned}
$$

(ii) Suppose $(1-\varepsilon)\left(\theta_{1}-\theta_{2}\right)<0$. There exists an SE where the followings hold:

$$
\begin{aligned}
& \frac{\dot{c}_{2, t}}{c_{2, t}}=\frac{\dot{h}_{2, t}}{h_{2, t}}=\frac{\dot{K}_{2, t}}{K_{2, t}}=\alpha A_{1}-\rho \equiv g^{*}, \\
& \frac{\dot{c}_{1, t}}{c_{1, t}}=\frac{\dot{h}_{1, t}}{h_{1, t}}=\frac{1+(\varepsilon-1) \theta_{2}}{1+(\varepsilon-1) \theta_{1}} g^{*} \equiv \hat{\hat{g}}^{*}\left(0<\hat{\hat{g}}^{*}<g^{*}\right), \\
& x_{1}=0\left(\equiv \hat{\hat{x}}_{1}^{*}\right), \eta_{1}=0\left(\equiv \hat{\hat{\eta}}_{1}^{*}\right), \eta_{2}=\frac{\phi_{2}\left[(1-\alpha) A_{1}+\rho\right]}{p\left(\phi_{2}+g^{*}\right)}\left(\equiv \hat{\hat{\eta}}_{2}^{*}\right), \\
& \lim _{t \rightarrow \infty} \frac{c_{1, t}}{h_{1, t}}=\lim _{t \rightarrow \infty} \frac{x_{t}}{\eta_{1, t}}=\frac{\phi_{1}+\hat{\hat{g}}^{*}}{\phi_{1}} .
\end{aligned}
$$

(Proof) See Appendix B

Proposition 3 provides many important implications. First, as shown in (20a) and (20b) (or (21a) and (21b)), when $\theta_{1} \neq \theta_{2}$ holds, growth is nonbalanced in the sense that the two sectors grow at the different (asymptotic) rates. We emphasize that because Proposition 1 holds, the aggregate economy exhibits balanced growth in the sense that $K_{t}$ and $E_{t}$ grow as the same rate.

In case of $(1-\varepsilon)\left(\theta_{1}-\theta_{2}\right)>0$, both consumption and output of the two goods grow at different rates in the SE. Output of Sector $i(=1,2)$ grows at the same rate of $K_{i, t}$. Note that $\hat{x}_{1}^{*}=(1-\alpha) A_{1}+\rho$ implies $x_{2, t}=0$ and $K_{2, t} / K_{t}=0$. Then, we have $K_{1, t} / K_{t}=1$ and hence $\dot{K}_{1, t} / K_{1, t}=g^{*}>\hat{g}^{*}=\dot{K}_{2, t} / K_{2, t}$ holds in the SE. Output of Sector 1 grows at a higher rate than that of Sector 2. When $\varepsilon<1$ holds, the condition for nonbalanced growth is $\theta_{1}>\theta_{2}$. When CUJ prevails, the sector producing the more positional good grows faster. Remember that when CUJ prevails, the growth rate of consumption of good $1\left(\dot{c}_{1, t} / c_{1, t}\right)$ is positively affected by the growth rate of the benchmark consumption of good $1\left(\dot{h}_{1, t} / h_{1, t}\right)$ and is negative affected by the growth rate of the benchmark consumption of good $2\left(\dot{h}_{2, t} / h_{2, t}\right)$ (see (12)). The positive effect increases with $\theta_{1}$ while the negative effect increases with $\theta_{2}$. Consequently, when $\theta_{1}>\theta_{2}$ holds, $c_{1, t}$ grows at a higher rate, which results in a higher growth in Sector 1. Then, nonbalanced growth of the two sectors arises.

Also in case of $(1-\varepsilon)\left(\theta_{1}-\theta_{2}\right)<0$, consumption of different goods grow at different rates in the SE. Consumption of good 2 grows at a higher rate than that of good 1. From 
the first equation of (21c), we know $x_{2, t}=(1-\alpha) A_{1}+\rho\left(=p c_{2, t} / K_{t}=A_{1} K_{2, t} / K_{t}\right)$, which implies $K_{1, t} / K_{t}=\alpha-\rho / A_{1}>0$ and $K_{2, t} / K_{t}=1-\alpha+\rho / A_{1}>0$. We then have $\dot{K}_{1, t} / K_{1, t}=$ $g^{*}=\dot{K}_{2, t} / K_{2, t}$. Outputs in the two sectors grow at the same rate in this equilibrium. Note that Sector 2 does not produce capital good. To meet the demand for good 2, firms in Sector 2 must increase capital input in the production of good 2. Because capital good is produced only in Sector 1, the demand of capital good for Sector 1 increases at the same rate as the demand for good 2. Consequently, outputs in the two sectors grow at the same rate.

Which is the more realistic parameter restriction, $(1-\varepsilon)\left(\theta_{1}-\theta_{2}\right)>0$ or $(1-\varepsilon)\left(\theta_{1}-\theta_{2}\right)<$ 0 ? As discussed in Subsection 2.2, we can reasonably consider good 1 that is used as capital good to be the more visible and positional good, and hence the case of $\theta_{1}>\theta_{2}$ is more relevant. Further, CUJ prevails in case of $\varepsilon<1$. The first case where $(1-\varepsilon)\left(\theta_{1}-\theta_{2}\right)>0$ holds is more realistic. Under this realistic case, both consumption and output of the two goods grow at different rates in the SE. Further, we know which sector grows at a higher rate. Under the reasonable parameter restriction, $\theta_{1}>\theta_{2}$ and $\varepsilon<1$, our results predict that consumption and output of the more positional good, good 1, grow at a higher rate than those of the less visible good.

Proposition 4 demonstrates that in case of $(1-\varepsilon)\left(\theta_{1}-\theta_{2}\right)>0, x_{2}\left(=(1-\alpha) A_{1}+\rho-x_{1}\right)$ tends to zero in the SE, which implies $K_{2, t} / K_{t}$ also tends to zero. However, this does not mean that Sector 2 does not produce anything because the economy approaches the SE only asymptotically. At all points in time, Sector 2 produces positive amounts and grows at a positive rate, $\hat{g}^{*}$. At the same time, output of Sector 2 relative to that of Sector 1 decreases because Sector 1 grow at a higher rate than Sector 2. In subsection 6.2, we examine the transitional dynamics with the help of phase diagrams. There, it will be observed that in case of $(1-\varepsilon)\left(\theta_{1}-\theta_{2}\right)>0$, shares of capital and labor allocated to Sector 2 gradually decreases along the transitional dynamics and hence the structural change occurs. Before we study the transitional dynamics, the next subsection examines the local stability of the SE.

\subsection{Stability}

We first consider the case of $(1-\varepsilon)\left(\theta_{1}-\theta_{2}\right)>0$. As in Acemoglu and Guerrieri (2008) who also examine the stability of the SE of a two-sector growth model where output share of a sector converge to zero, we linearize the dynamic system around the SE characterized by (20a)-(20d).

$$
\left(\begin{array}{c}
\dot{x}_{1, t} \\
\dot{\eta}_{1, t} \\
\dot{\eta}_{2, t}
\end{array}\right)=\Omega\left(\begin{array}{c}
x_{1, t}-\hat{x}_{1}^{*} \\
\eta_{1, t}-\hat{\eta}_{1}^{*} \\
\eta_{2, t}-\hat{\eta}_{2}^{*}
\end{array}\right),
$$

where the Jacobian matrix, $\Omega$, is given by:

$$
\Omega=\left(\begin{array}{ccc}
-(1-\varepsilon)\left(\theta_{1} g^{*}-\theta_{2} \hat{g}^{*}\right)+(1-\varepsilon) \theta_{2}\left(\phi_{2}+\hat{g}^{*}\right) & 0 & \frac{(1-\varepsilon) p \theta_{2}}{\phi_{2}}\left(\phi_{2}+\hat{g}^{*}\right)^{2} \\
\phi_{1} & -\left(\phi_{1}+g^{*}\right) & 0 \\
-\phi_{2} / p & 0 & -\left(\phi_{2}+g^{*}\right)
\end{array}\right) .
$$


In deriving $\Omega$, we use (20a)-(20d). Appendix $C$ shows that the eigenvalues of $\Omega$ are given by:

$$
\begin{aligned}
& \mu_{1}=-\left(\phi_{1}+g^{*}\right)(<0), \\
& \mu_{2}=-(1-\varepsilon)\left(\theta_{1} g^{*}-\theta_{2} \hat{g}^{*}\right)(<0), \\
& \mu_{3}=-\left\{1+(\varepsilon-1) \theta_{2}\right\} \phi_{2}-g^{*}-(\varepsilon-1) \theta_{2} \hat{g}^{*}(<0) .
\end{aligned}
$$

It is shown that $\mu_{2}$ and $\mu_{3}$ are both negative. In sum, the three eigenvalues of $\Omega$ are negative. Remember that we should treat $x_{1,0}$ as a predetermined variable and that $\eta_{1}$ and $\eta_{2}$ are also non-jumpable variables. Because there are three stable roots, the SE is locally stable.

We next examine the stability of the SE in case of $(1-\varepsilon)\left(\theta_{1}-\theta_{2}\right)<0$. Again, we linearize the dynamic system around the SE characterized by (21a)-(21d).

$$
\left(\begin{array}{c}
\dot{x}_{1, t} \\
\dot{\eta}_{1, t} \\
\dot{\eta}_{2, t}
\end{array}\right)=\Gamma\left(\begin{array}{c}
x_{1, t}-\hat{\hat{x}}_{1}^{*} \\
\eta_{1, t}-\hat{\hat{\eta}}_{1}^{*} \\
\eta_{2, t}-\hat{\hat{\eta}}_{2}^{*}
\end{array}\right),
$$

where the Jacobian matrix, $\Gamma$, is given by:

$$
\Gamma=\left(\begin{array}{ccc}
(1-\varepsilon)\left(\theta_{1} \hat{\hat{g}}^{*}-\theta_{2} g^{*}\right)+(1-\varepsilon) \theta_{2}\left(\phi_{2}+\hat{\hat{g}}^{*}\right) & \frac{(1-\varepsilon) \theta_{1}}{\phi_{1}}\left(\phi_{1}+\hat{g}^{*}\right)^{2} & 0 \\
\phi_{1} & -\left(\phi_{1}+g^{*}\right) & 0 \\
-\phi_{2} / p & 0 & -\left(\phi_{2}+g^{*}\right)
\end{array}\right) .
$$

In deriving $\Gamma$, we use (21a)-(21d). Appendix D shows that the eigenvalues of $\Gamma$ are given by:

$$
\begin{aligned}
& \zeta_{1}=-\left(\phi_{2}+g^{*}\right)(<0), \\
& \zeta_{2}=(1-\varepsilon)\left(\theta_{1} \hat{\hat{g}}^{*}-\theta_{2} g^{*}\right)(<0), \\
& \zeta_{3}=-\left\{1+(\varepsilon-1) \theta_{1}\right\} \phi_{1}-g^{*}-(\varepsilon-1) \theta_{1} \hat{\hat{g}}^{*}(<0) .
\end{aligned}
$$

It is shown that $\zeta_{2}$ and $\zeta_{3}$ are both negative. In sum, the three eigenvalues of $\Gamma$ are negative, so that the SE satisfies local stability. The next proposition summarizes the results obtained so far.

\section{Proposition 5}

If $\varepsilon \neq 1$ and $\theta_{1} \neq \theta_{2}$, then the SEs we consider are locally stable.

\subsection{Transitional Dynamics}

Subsection 6.1 shows the stability of the SE. This section observes how structural change progresses along the transition to the SE with the help of phase diagram. We focus on the case where $\varepsilon<1$ and $\theta_{1}>\theta_{2}$ hold. For simplicity, we assume $\theta_{1}>0=\theta_{2}$.

The following three lines consist of $\dot{x}_{1, t}=0$ locus:

$$
x_{1}=0, \quad x_{1}=(1-\alpha) A_{1}+\rho, \quad \eta_{1}=x_{1} .
$$


Note that $0 \leq x_{1} \leq(1-\alpha) A_{1}+\rho$ must hold from (15). Hence, we restrict our attention to the region where this relationship holds. As shown in Figure 3, we have $\dot{x}_{1}<(>) 0$ in the region above (below) the graph of $\eta_{1}=x_{1}$. From (16b), we obtain $\dot{\eta}_{1}=0$ locus

$$
\eta_{1}=\frac{\phi_{1}}{\phi_{1}+g^{*}} x_{1} .
$$

Because $g^{*}$ is strictly positive, the slope of $\dot{\eta}_{1}=0$ locus is less steeper than that of $\eta_{1}=x_{1}$. We have $\dot{\eta}<(>) 0$ in the region above (below) $\dot{\eta}_{1}=0$ locus.

The initial value of $\eta_{1, t}\left(\equiv h_{1, t} / K_{t}\right)$ is determined by $K_{0}$ and $h_{1,0}$. From (17), the initial value of $x_{1, t}$ is given by:

$$
x_{1,0}=\frac{(1-\alpha) A_{1}+\rho}{1+p z h_{1,0} \theta_{1}(\varepsilon-1)}=\frac{(1-\alpha) A_{1}+\rho}{1+p z \eta_{1,0} \theta_{1}^{(\varepsilon-1)} K_{0}^{\theta_{1}(\varepsilon-1)}} .
$$

Given $K_{0}$, both $x_{1,0}$ and $\eta_{1,0}$ increase with $h_{1,0}$. In Figure 3, which illustrates the phase diagram in $\left(x_{1}, \eta_{1}\right)$ space, given $K_{0}$, we plot three initial points. Among three points, $h_{1,0}$ is smallest in Point A and largest in Point $\mathrm{C}$.

If the economy is initially located on Point A, the economy monotonically converges to the SE. Along the transition, $x_{1, t}$ monotonically increases to its SE value. Note that expenditure shares of good 1 and 2 are given by $c_{1, t} / E_{t}=x_{1, t} /\left\{(1-\alpha) A_{1}+\rho\right\}$ and $p c_{2, t} / E_{t}=$ $1-x_{1, t} /\left\{(1-\alpha) A_{1}+\rho\right\}$ and production shares of good 1 and 2 are given by $Y_{1, t} / Y_{t}=\alpha A_{1}-\rho+$ $x_{1, t}$ and $Y_{2, t} / Y_{t}=(1-\alpha) A_{1}+\rho-x_{1, t}$. Along the transition to the SE, expenditure and output shares of good 1 monotonically increase while those of good 2 monotonically decrease. Because the production of Sector $i$ is equal to $A_{i} K_{i}(i=1,2)$, the share of capital allocated to Sector 1 increases while the share of capital allocated to Sector 2 decreases along the transition. From (1b), we have $l_{2, t} / l_{1, t}=K_{2, t} / K_{1, t}$, which indicates that labor allocated to Sector 1 also increases while labor allocated to Sector 2 also decreases. Then, structural change steadily progresses along the transition. We emphasize that while structural change progresses, the aggregate economy exhibits balanced growth in the sense that $K_{t}$ and $E_{t}$ grow at the same constant rate because Proposition 1 holds.

Figure 3 provides the further implication. From Proposition 1, we know that if there are two countries that have the same technology and the same preference, the growth rates of outputs in the two countries become exactly the same. If the initial values of $K_{t}$ are the same in addition, the output levels of the two countries also become exactly the same over time and hence the two countries exhibit exactly the same performance of aggregate economy. However, if the initial values of $h_{1, t}$ are different, the two countries follow the different patterns of structural change. Consequently, the two countries have different expenditure and production shares of the two goods, and different shares of capital and labor allocated to the two sectors at all points in time although these two countries have exactly the same performance of the aggregate economy. Our model suggests that the presence of consumption externalities might be a source of the divergent patterns of structural change across countries.

We finally show that the dependence of the benchmark consumption levels on the past consumption is crucial for the divergent patterns of structural change across countries. Consider the case of $\phi_{1}=+\infty$ where the consumption externalities are only intratemporal. In 
equilibrium, we have $h_{1, t}=c_{1, t}$ and $\eta_{1, t}=x_{1, t}$. From (12), (13) and (14), we obtain:

$$
\dot{x}_{1, t}=\frac{g^{*}(1-\varepsilon) \theta_{1}\left\{1-\frac{x_{1, t}}{(1-\alpha) A_{1}+\rho}\right\}}{1-(1-\varepsilon) \theta_{1}\left\{1-\frac{x_{1, t}}{(1-\alpha) A_{1}+\rho}\right\}} x_{1, t} .
$$

Figure 4 presents the graph of the above equation. (24) can be written as:

$$
x_{1,0}=\frac{(1-\alpha) A_{1}+\rho}{1+p z x_{1,0}^{\theta_{1}(\varepsilon-1)} K_{0}^{\theta_{1}(\varepsilon-1)}},
$$

which shows that two countries endowed with the same level of $K_{0}$ have the same initial value of $x_{1, t}$. Then, from Figure 4, we know that in the case of $\phi_{1}=+\infty$, the two countries that exhibit exactly the same performance of aggregate economy follow exactly the same pattern of structural change.

[Figure 4]

\section{Conclusion}

This paper has demonstrated the important role of commodity-specific consumption externalities in generating structural change using a two-sector general equilibrium model. Under the presence of commodity-specific consumption externalities, the utility function exhibits non-homothetic from the perspective of social consumption, even though the individual's preference is homothetic with respect to each good and production function of each sector is of the AK form with constant productivity. We have shown not only that structural change occurs when the degrees of consumption externalities are different from each other, but also that aggregate economy exhibits balanced growth in the sense that capital stock and expenditure grow at the same constant rate, which is consistent with the Kaldor facts.

Our results have shown the important implications for differences in country pattens of structural change. If the two countries have different initial benchmark levels of consumption goods, they follow the different patterns of structural change despite the same initial values of capital stock, technologies and preferences. Furthermore, their aggregate economy exhibit exactly the same performance. This could be a possible explanation for why the patterns of structural change vary across structurally similar countries (Jorgenson and Timmer, 2011).

We have restricted ourselves to the case where the intertemporal elasticity of substitution in consumption is equal to one. A straightforward extension of the current model is to relax this restriction. The feature of the dynamics in our economy strongly depend on the choice of the intertemporal elasticity of substitution in consumption. Also, the stability in an equilibrium could be affected by a change in the intertemporal elasticity of substitution in consumption under the presence of commodity-specific consumption externalities.

As for another possible extensions of our investigation, we may consider a three-sector model. In this paper, we focus on the role of commodity-specific consumption externalities in generating structural change. We have shown that the two sector grow at the different 
rate in the equilibrium and either sector always grows faster than the other, depending on parameter values. It is interesting to pursue further studies on the hump-shaped share of manufacturing observed in the data. Employing a three-sector model would yield nonmonotonic reallocation of resources in the course of structural change.

\section{Appendix}

\section{A The Derivation of (12)}

This appendix presents the derivations of (12). By differentiating (4) with respect to $t$, we obtain:

$$
\frac{\dot{u}_{t}}{u_{t}}=u_{t} \frac{1-\varepsilon}{\varepsilon}\left\{\gamma\left(c_{1, t} h_{1, t}{ }^{-\theta_{1}}\right)^{\frac{\varepsilon-1}{\varepsilon}}\left(\frac{\dot{c}_{1, t}}{c_{1, t}}-\theta_{1} \frac{\dot{h}_{1, t}}{h_{1, t}}\right)+(1-\gamma)\left(c_{2, t} h_{2, t}{ }^{-\theta_{2}}\right)^{\frac{\varepsilon-1}{\varepsilon}}\left(\frac{\dot{c}_{2, t}}{c_{2, t}}-\theta_{1} \frac{\dot{h}_{2, t}}{h_{2, t}}\right)\right\} .
$$

Using (4) and (11) in (A.1), we obtain:

$$
\frac{\dot{u}_{t}}{u_{t}}=\frac{\dot{c}_{1, t}}{c_{1, t}}-\theta_{1} \frac{\dot{h}_{1, t}}{h_{1, t}}+\varepsilon \frac{(1-\gamma)\left(c_{2, t} h_{2, t}{ }^{-\theta_{2}}\right)^{\frac{\varepsilon-1}{\varepsilon}}}{u^{\frac{\varepsilon-1}{\varepsilon}}}\left(\theta_{1} \frac{\dot{h}_{1, t}}{h_{1, t}}-\theta_{2} \frac{\dot{h}_{2, t}}{h_{2, t}}\right) .
$$

From (4), we have $u_{c_{1, t}}=\gamma u^{1 / \varepsilon}\left(c_{1, t} h_{1, t}{ }^{-\theta_{1}}\right)^{\frac{\varepsilon-1}{\varepsilon}} c_{1, t}^{-1}$ where $u_{c_{1, t}}$ is the partial derivative of $u$ with respect to $c_{1, t}$. Differentiating both sides of this equation with respect to $t$ yields:

$$
\frac{\dot{u}_{c_{1, t}}}{u_{c_{1, t}}}-\frac{\dot{u}_{t}}{u_{t}}=\frac{1-\varepsilon}{\varepsilon} \frac{\dot{u}_{t}}{u_{t}}-\frac{1}{\varepsilon} \frac{\dot{c}_{1, t}}{c_{1, t}}-\theta_{1} \frac{\varepsilon-1}{\varepsilon} \frac{\dot{h}_{1, t}}{h_{1, t}} .
$$

Using (7b) and the subutility function, we obtain:

$$
\frac{u_{c_{1, t}}}{u}=\frac{\partial \ln u}{\partial c_{1}}=\frac{\partial \ln u}{\partial E} \frac{\partial E}{\partial c_{1}}=\frac{1}{E} .
$$

From the above equation and (10), we know that the left-hand side of (A.3) is equal to $\rho-\alpha A_{1}$. Therefore, from (A.2) and (A.3), we obtain:

$$
\frac{\dot{c}_{1, t}}{c_{1, t}}=\alpha A_{1}-\rho+(1-\varepsilon) \frac{(1-\gamma)\left(c_{2, t} h_{2, t}{ }^{-\theta_{2}}\right)^{\frac{\varepsilon-1}{\varepsilon}}}{u^{\frac{\varepsilon-1}{\varepsilon}}}\left(\theta_{1} \frac{\dot{h}_{1, t}}{h_{1, t}}-\theta_{2} \frac{\dot{h}_{2, t}}{h_{2, t}}\right) .
$$

We rearrange (8) as follows:

$$
\frac{1-\gamma}{\gamma}\left(\frac{c_{2, t} h_{2, t}{ }^{-\theta_{2}}}{c_{1, t} h_{1, t}{ }^{-\theta_{1}}}\right)^{\frac{\varepsilon-1}{\varepsilon}}=\frac{p c_{2, t}}{c_{1, t}}
$$

By using (4) and (A.5), we have:

$$
\frac{(1-\gamma)\left(c_{2, t} h_{2, t}{ }^{-\theta_{2}}\right)^{\frac{\varepsilon-1}{\varepsilon}}}{u^{\frac{\varepsilon-1}{\varepsilon}}}=\frac{\frac{1-\gamma}{\gamma}\left(\frac{c_{2, t} h_{2, t}{ }^{-\theta_{2}}}{c_{1, t} h_{1, t}{ }^{-\theta_{1}}}\right)^{\frac{\varepsilon-1}{\varepsilon}}}{1+\frac{1-\gamma}{\gamma}\left(\frac{c_{2, t} h_{2, t}{ }^{-\theta_{2}}}{c_{1, t} h_{1, t}}\right)^{\frac{\varepsilon-1}{\varepsilon}}}=\frac{p c_{2, t}}{c_{1, t}+p c_{2, t}}=1-\frac{c_{1, t}}{E_{t}} .
$$

By substituting these two equations into (A.4), we can derive (12). 


\section{B Proof of Proposition 4}

We first prove the case of $(1-\varepsilon)\left(\theta_{1}-\theta_{2}\right)>0$. By substituting equations in (20c) into (16a)-(16c), we obtain $\dot{x}_{1, t}=\dot{\eta}_{1, t}=\dot{\eta}_{2, t}=0$. Values in (20c) are the candidate of an SE. Because $\dot{x}_{1, t}=\dot{\eta}_{1, t}=0$ and both $\hat{x}_{1}^{*}$ and $\hat{\eta}_{1}^{*}$ are strictly positive, we obtain (20a). From $\dot{c}_{2, t} / c_{2, t}=\dot{h}_{2, t} / h_{2, t},(11)$ and (20a), we obtain (20b). Because of $1+(\varepsilon-1) \theta_{i}>0(i=1,2)$, we have $\hat{g}^{*}>0$. The inequality $(1-\varepsilon)\left(\theta_{1}-\theta_{2}\right)>0$ ensures $\hat{g}^{*}<g^{*}$, which is consistent with $\hat{\eta}_{2}^{*}=0$. From (16c) and (20b), we obtain (20d).

We turn to the case of $(1-\varepsilon)\left(\theta_{1}-\theta_{2}\right)<0$. By substituting equations in (21c) into (16a)-(16c), we obtain $\dot{x}_{1, t}=\dot{\eta}_{1, t}=\dot{\eta}_{2, t}=0$. Values in (21c) are the candidate of an SE. Because of $\hat{x}_{1}^{*}=0$, we have $x_{2}=(1-\alpha) A_{1}+\rho>0$, which implies $\dot{c}_{2, t} / c_{2, t}=g^{*}$. In addition, because $\dot{\eta}_{2, t}=0$ and $\hat{\eta}_{2}^{*}$ is strictly positive, we obtain (21a). From $\dot{c}_{1, t} / c_{1, t}=\dot{h}_{1, t} / h_{1, t}$, (11) and (21a), we obtain $(21 \mathrm{~b})$. Because of $1+(\varepsilon-1) \theta_{i}>0(i=1,2)$, we have $\hat{\hat{g}}^{*}>0$. The inequality $(1-\varepsilon)\left(\theta_{1}-\theta_{2}\right)>0$ ensures $\hat{\hat{g}}^{*}<g^{*}$, which is consistent with $\hat{\eta}_{1}^{*}=0$. From (16b) and (21b), we obtain (21d).

\section{The Eigenvalues of $\Omega$}

One of the eigenvalues of $\Omega$ is apparently $\mu_{1}=-\left(\phi_{1}+g^{*}\right)$. The other two eigenvalues, $\mu_{2}$ and $\mu_{3}$, satisfy:

$$
\begin{aligned}
\mu_{2}+\mu_{3} & =-(1-\varepsilon)\left(\theta_{1} g^{*}-\theta_{2} \hat{g}^{*}\right)-\left\{1+(\varepsilon-1) \theta_{2}\right\} \phi_{2}-g^{*}-(\varepsilon-1) \theta_{2} \hat{g}^{*} \\
\mu_{2} \mu_{3} & =(1-\varepsilon)\left(\theta_{1} g^{*}-\theta_{2} \hat{g}^{*}\right)\left(\phi_{2}+g^{*}\right)-(1-\varepsilon) \theta_{2}\left(\phi_{2}+\hat{g}^{*}\right)\left(g^{*}-\hat{g}^{*}\right) \\
& =\left[-(1-\varepsilon)\left(\theta_{1} g^{*}-\theta_{2} \hat{g}^{*}\right)\right]\left[-\left\{1+(\varepsilon-1) \theta_{2}\right\} \phi_{2}-g^{*}-(\varepsilon-1) \theta_{2} \hat{g}^{*}\right] .
\end{aligned}
$$

Then, the eigenvalues of $\Omega$ are given by (22a)-(22c).

We next check the signs of $\mu_{2}$ and $\mu_{3}$. By using the definition of $\hat{g}^{*}$, we can rewrite $\mu_{2}$ as follows:

$$
\mu_{2}=-\frac{(1-\varepsilon)\left(\theta_{1}-\theta_{2}\right)}{1+(\varepsilon-1) \theta_{2}} g^{*} .
$$

We now consider the case of $(1-\varepsilon)\left(\theta_{1}-\theta_{2}\right)>0$. And we have $1+(\varepsilon-1) \theta_{2}>1-\theta_{2}>0$ because of $\varepsilon>0$ and $0<\theta_{2}<1$. Hence, $\mu_{2}$ is negative. We can show that $\mu_{3}$ is negative in the following way:

$$
\begin{aligned}
\mu_{3} & <-\left\{1+(\varepsilon-1) \theta_{2}\right\}\left(\phi_{2}+\hat{g}^{*}\right) \\
& <-\left(1-\theta_{2}\right)\left(\phi_{2}+\hat{g}^{*}\right) \\
& <0 .
\end{aligned}
$$

The first inequality holds because of $\hat{g}^{*}<g^{*}$. The inequalities $\varepsilon>0$ and $\theta_{2}>0$ ensure the second inequality. The last inequality holds because of $\theta_{2}<1$. 


\section{The Eigenvalues of $\Gamma$}

One of the eigenvalues of $\Gamma$ is apparently $\zeta_{1}=-\left(\phi_{2}+g^{*}\right)$. The other two eigenvalues, $\zeta_{2}$ and $\zeta_{3}$, satisfy:

$$
\begin{aligned}
\zeta_{2}+\zeta_{3} & =(1-\varepsilon)\left(\theta_{1} \hat{\hat{g}}^{*}-\theta_{2} g^{*}\right)-\left\{1+(\varepsilon-1) \theta_{1}\right\} \phi_{1}-g^{*}-(\varepsilon-1) \theta_{1} \hat{\hat{g}}^{*}, \\
\zeta_{2} \zeta_{3} & =-(1-\varepsilon)\left(\theta_{1} \hat{\hat{g}}^{*}-\theta_{2} g^{*}\right)\left(\phi_{1}+g^{*}\right)+(1-\varepsilon) \theta_{1}\left(\phi_{1}+\hat{\hat{g}}^{*}\right)\left(\hat{\hat{g}}^{*}-g^{*}\right) \\
& \left.=\left[(1-\varepsilon)\left(\theta_{1} \hat{\hat{g}}^{*}-\theta_{2} g^{*}\right)\right]\left[-\theta_{2} g^{*}\right)-\left\{1+(\varepsilon-1) \theta_{1}\right\} \phi_{1}-g^{*}-(\varepsilon-1) \theta_{1} \hat{\hat{g}}^{*}\right] .
\end{aligned}
$$

Then, the eigenvalues of $\Gamma$ are given by (23a)-(23c).

We next check the signs of $\zeta_{2}$ and $\zeta_{3}$. By using the definition of $\hat{\hat{g}}^{*}$, we can rewrite $\zeta_{2}$ as follows:

$$
\zeta_{2}=\frac{(1-\varepsilon)\left(\theta_{1}-\theta_{2}\right)}{1+(\varepsilon-1) \theta_{2}} g^{*}
$$

Now consider the case of $(1-\varepsilon)\left(\theta_{1}-\theta_{2}\right)<0$. And we have $1+(\varepsilon-1) \theta_{2}>1-\theta_{2}>0$ because of $\varepsilon>0$ and $0<\theta_{2}<1$. Hence, $\zeta_{2}$ is negative. It is also shown that $\zeta_{3}<0$ because

$$
\begin{aligned}
\zeta_{3} & <-\left\{1+(\varepsilon-1) \theta_{1}\right\}\left(\phi_{1}+\hat{\hat{g}}^{*}\right) \\
& <-\left(1-\theta_{1}\right)\left(\phi_{1}+\hat{\hat{g}}^{*}\right) \\
& <0 .
\end{aligned}
$$

The first inequality holds because of $\hat{g}^{*}<g^{*}$. The inequalities $\varepsilon>0$ and $\theta_{2}>0$ ensure the second inequality. The last inequality holds because of $\theta_{2}<1$. 


\section{References}

Acemoglu, Daron. and Veronica Guerrieri (2008) "Capital deepening and nonbalanced economic growth", Journal of Political Economy, 116, 467-498.

Alpizar, Francisco., Fredrik Carlsson, and Olof Johansson-Stenman (2005) "How much do we care about absolute versus relative income and consumption?" Journal of Economic Behavior E Organization, 56, 405-421.

Buera, Francisco.J. and Joseph P. Kaboski (2009) "Can traditional theories of structural change fit the data?", Journal of the European Economic Association, 7, 469-477.

Baumol, William J. (1967) "Macroeconomics of unbalanced growth: the anatomy of urban crisis", American Economic Review, 57, 415-426.

Carlsson, Fredrik., Olof Johansson-Stenman and Peter Martinsson (2007) "Do You Enjoy Having More than Others? Survey Evidence of Positional Goods", Economica, 74, 586598.

Carroll, Christopher D., Jody Overland., and David N. Weil. (1997) "Comparison Utility in a Growth Model”, Journal of Economic Growth, 2, 339-367.

(2000) "Savings and Growth with Habit Formation", American Economic Review, 90, 341-355.

Doi, Junko and Kazuo Mino (2008) "A Variety Expansion Model of Growth with External Habit," Journal of Economic Dynamics $\mathcal{E}$ Control, 32, 3055-3083.

Foellmi, Reto and Josef Zweimüller (2008) "Structural change, Engel's consumption cycles and Kaldor's facts of economic growth", Journal of Monetary Economics, 55, 13171328 .

Guill(o, Mar'ia D., Chris Papageorgiou, and Fidel Perez-Sebastian (2011) "A unified theory of structural change," Journal of Economic Dynamics E Control, 35, 1393-1404.

Hori, Takeo (2011) "The Effects of Consumption Externalities in an R\&D-Based Growth Model with Endogenous Skilled and Unskilled Labor Supply”, Journal of Economics, $102,29-55$.

Jorgenson, Dale W. and Marcel P. Timmer (2011) "Structural change in advanced nations: a new set of stylised facts" Scandinavian Journal of Economics, 113, 1-29.

Kongsamut, Piyabha., Sergio Rebelo and Danyang Xie (2001) "Beyond balanced growth", Review of Economic Studies, 68, 869-882.

Ngai, L.R. and C.A. Pissarides (2007) "Structural change in a multisector model of growth", American Economic Review, 97, 429-445. 
Ravn, Morten, Stephanie Schmitt-Grohé, and Mart'in Uribe (2006) "Deep Habits," Review of Economic Studies, 73, 195-218.

Solnick, Sara J. and David Hemenway (2001) "Is more always better? A survey on positional concerns", Journal of Economic Behavior E Organization, 37, 373-383. 


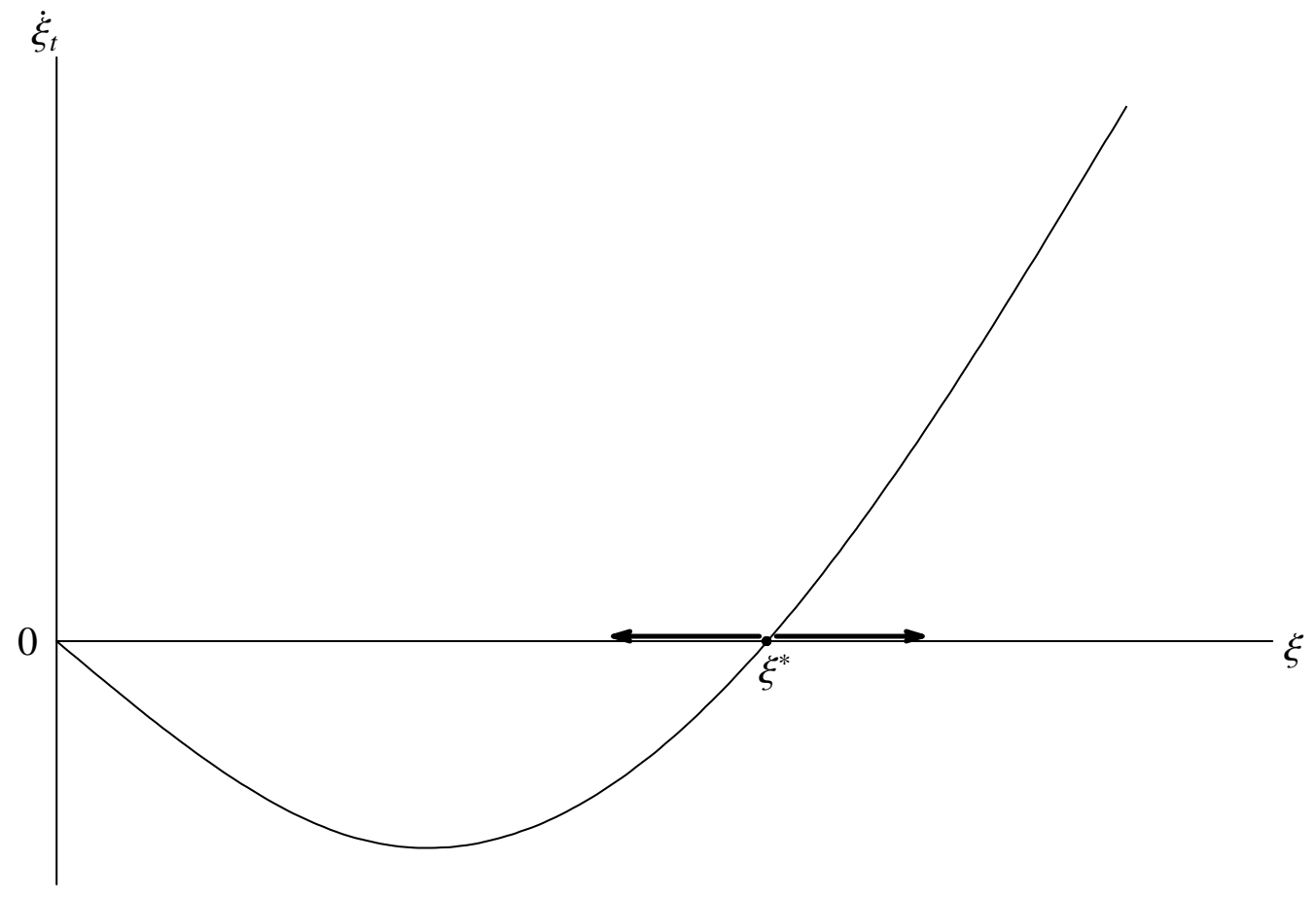

Figure 1. Dynamics of $\xi$ 

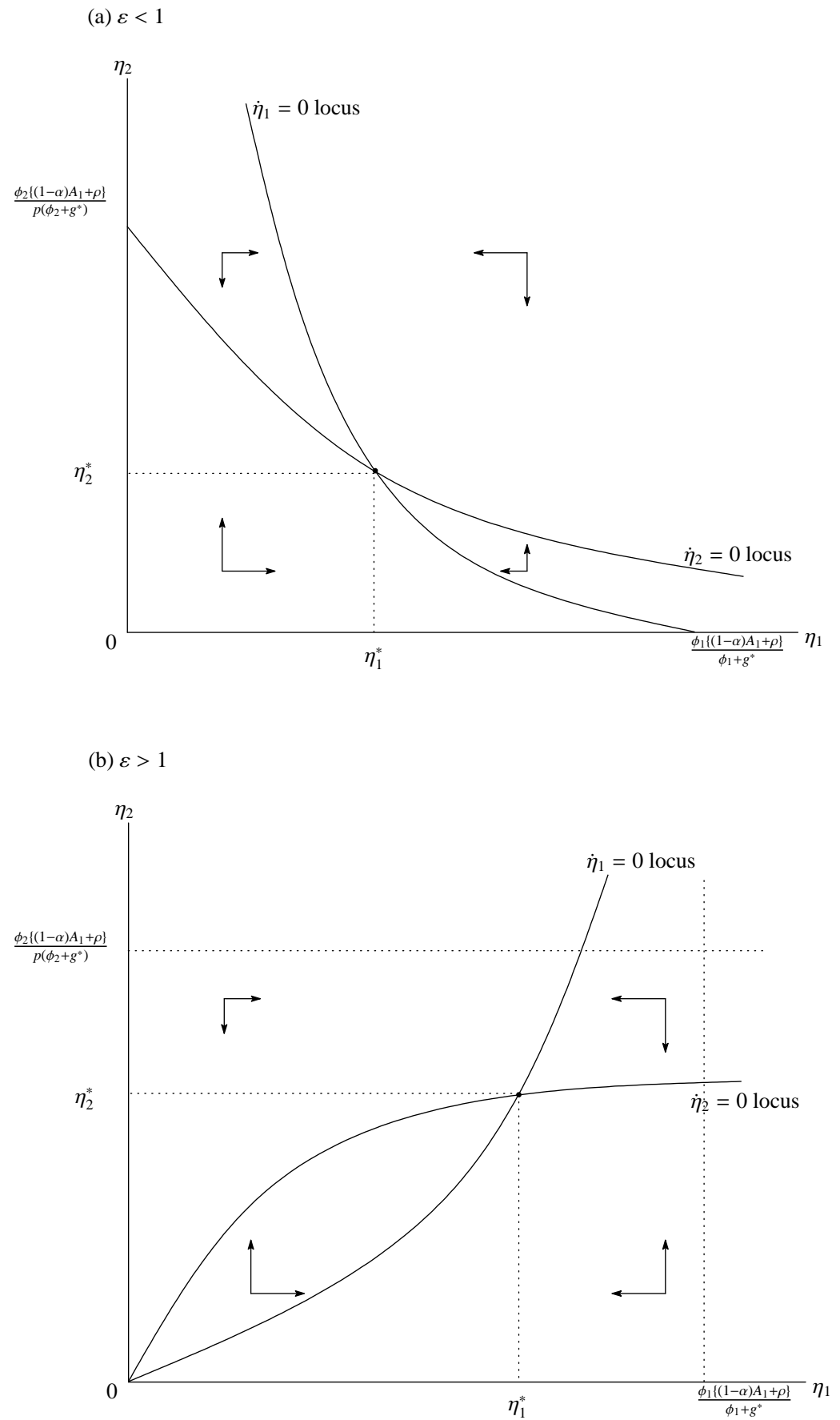

Figure 2. Phase Diagrams: $\theta_{1}=\theta_{2}$ 


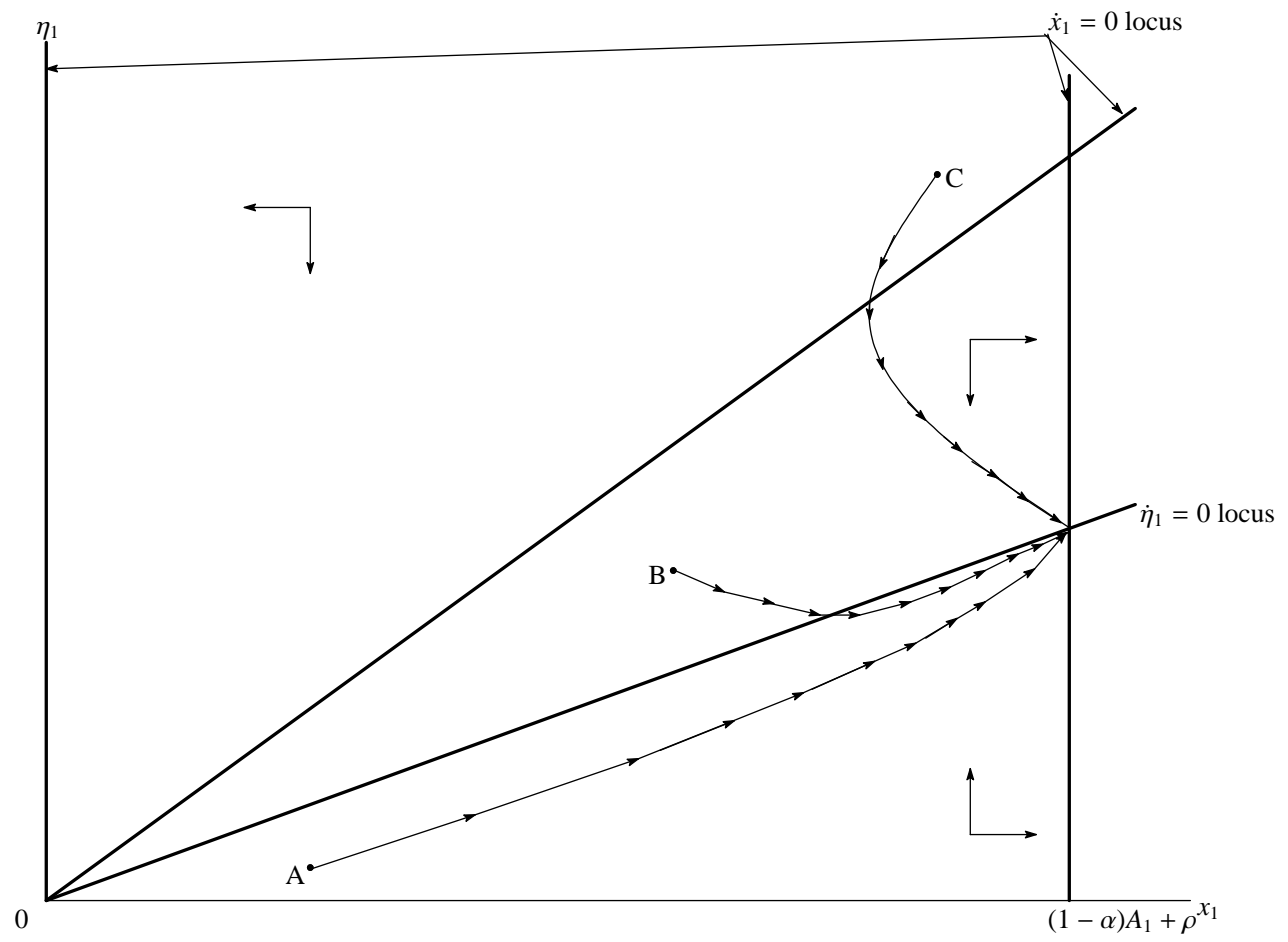

Figure 3. Phase Diagrams: $\varepsilon<1$ and $\theta_{1}>0=\theta_{2}$ 


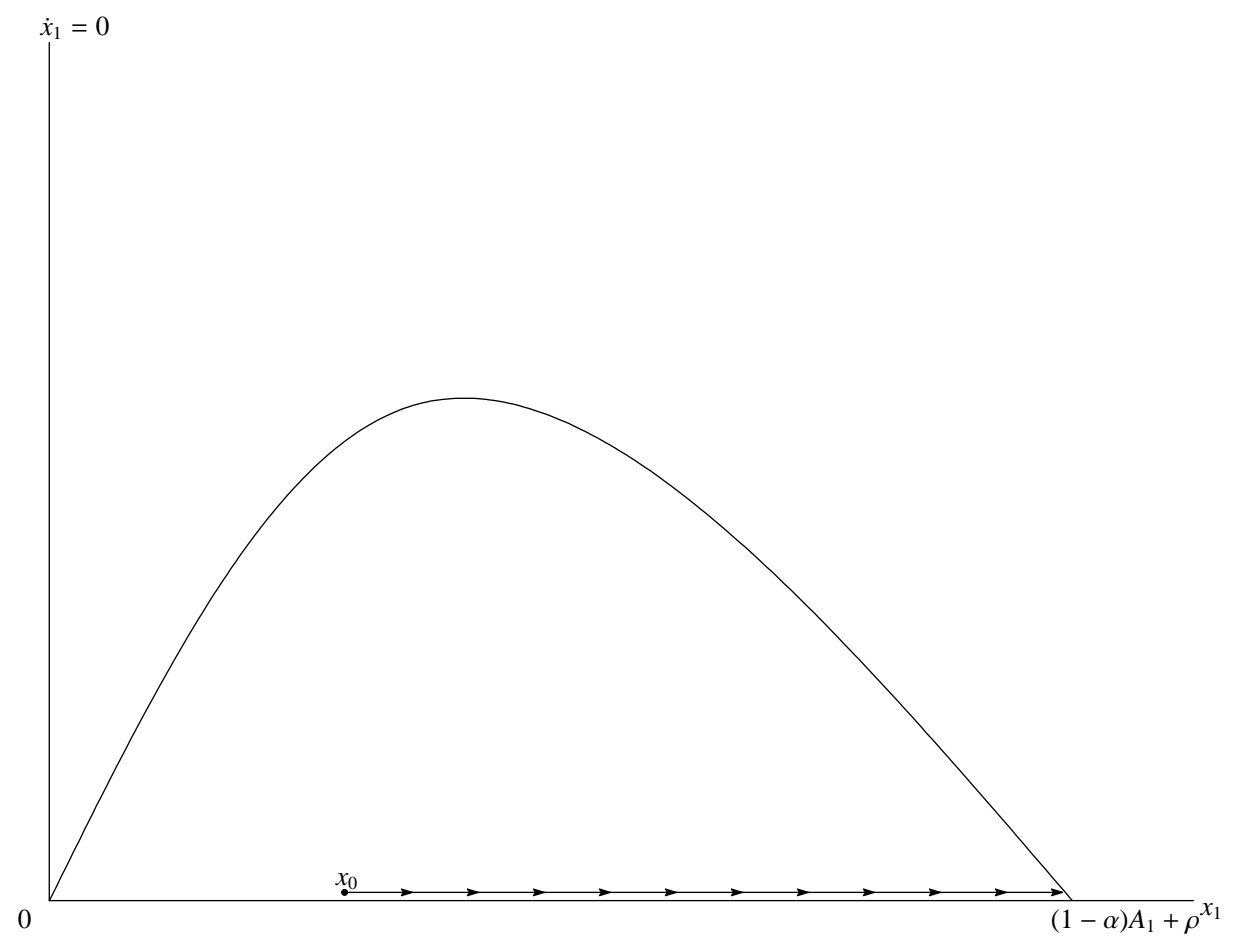

Figure 4. Phase Diagrams: $\varepsilon<1, \theta_{1}>0=\theta_{2}$ and $\phi_{1}=+\infty$ 